\title{
Improved protein arrays for quantitative systems analysis of the dynamics of signaling pathway interactions
}

\author{
Xiaoyu Wang ${ }^{1,2}$, Ying Dong ${ }^{1,3}$, Ameena J Jiwani 1,5,6, Yonglong Zou ${ }^{1,3}$, Johanne Pastor ${ }^{4}$, Makoto Kuro-o ${ }^{1,4}$, \\ Amyn A Habib ${ }^{1,5,6^{*}}$, Minzi Ruan ${ }^{7 *}$, David A Boothman ${ }^{1,3}$ and Chin-Rang Yang ${ }^{1,2^{*}}$
}

\begin{abstract}
An improved version of quantitative protein array platform utilizing linear Quantum dot signaling for systematically measuring protein levels and phosphorylation states is presented. The signals are amplified linearly by a confocal laser Quantum dot scanner resulting in $~ 1000$-fold more sensitivity than traditional Western blots, but are not linear by the enzyme-based amplification. Software is developed to facilitate the quantitative readouts of signaling network activities. Kinetics of EGFRvIll mutant signaling was analyzed to quantify cross-talks between EGFR and other signaling pathways.
\end{abstract}

Keywords: protein array, quantum dot, quantitative analysis, signaling pathway, cross-talk, image analysis, glioma, systems biology

\section{Background}

The emerging field of Systems Biology focuses on the most daunting challenges in biology and medicine. It is an attempt to understand how all parts of the cell - genes, proteins, and other molecules - work in concert to create complex living organisms and analyzing how entire biological systems function, both in health and in sickness. Systems biology always relates big amount of data, which makes high throughput technologies are crucial. The rapid advance of high throughput technologies has enabled scientists to broaden their research from detailed investigation of a few selected genes/proteins to global gene/protein expression profiles and network analysis. Among the network analysis, cellular signal transduction networks play an important role in regulating cellular processes, such as proliferation, cell growth and death. Proteins are the work-horses that carry out these functions. Therefore, it is crucial to capture the dynamics of protein kinases and post-translational regulations within cellular signal transduction networks for understanding how the signaling

\footnotetext{
* Correspondence: amyn.habib@utsouthwestern.edu; minzi@vigenetech.com; chinrang.yang@utsouthwestern.edu

${ }^{1}$ Harold C. Simmons Comprehensive Cancer Center, University of Texas Southwestern Medical Center at Dallas, Dallas, TX 75390, USA

${ }^{7}$ VigeneTech, Inc. Carlisle, MA 01741, USA

Full list of author information is available at the end of the article
}

pathways are operated in healthy versus disease conditions.

Reverse phase protein lysate array (RPPA), originally introduced by Drs L. Liotta and E. Petricoin [1], is designed for measuring protein expression in a large number of biological samples quantitatively. Sample lysates were spotted in series of dilutions to generate dilution curves for quantitative measurements. Arrays are probed with a primary antibody followed by a speciesspecific secondary antibody similar to the Western blot. The detection signal comes from the tag on the secondary antibody. A range of detection tags have been developed including colorimetric, fluorescent, near-infrared (IRDye), and Quantum dot (Qdot) assays [2-6]. RPPA has been applied to protein monitoring for biomarker discovery and/or signal transduction proteins in response to various biological stimuli or chemical treatments [7-10]. However, to use RPPA as a quantification assay is a real challenge, because the linear signals, the foundation of quantification, are difficult to be obtained by using the common enzyme-based (horseradish peroxidase, HRP) signal amplification systems such as Tyramide Signal Amplification (TSA ${ }^{\mathrm{TM}}$, Molecular Probes), or Catalyzed Signal Amplification (CSATM, Dako) [2-5]. Non-enzyme based signal detection based on IRDye with 
Odyssey scanner (LI-COR) [11] as well as Qdot with hyperspectral imaging microscope (not commercial available) [6] have been reported. Here, we report another alternative non-enzyme amplification approach using Qdot and commercial available confocal laser Qdot scanner for protein quantification.

The Qdot is a nano-metal fluorophore with bright and linear signal, and the advantage of using Qdot is it has no photo-bleaching effect that often occurs while using organic fluorophores. In combination of confocal laser Qdot scanner, we present an enhanced version of the RPPA platform for sensitive, reproducible and quantitative cellular signal transduction network measurements. The cell lysis buffer is optimized for RPPA printing and dissolving whole cell proteins without using urea. The thin-coated-nitrocellulose slide is chosen for strong protein binding and low fluorescence background. A confocal laser Qdot scanner is utilized to amplify and maintain the signal linearity. The widely used enzyme-based amplification is not linear, resulting in nonlinearity signals that not suitable for the quantification is also demonstrated. To further reduce background fluorescence from nitrocellulose and increase signal/noise ratios, the advantage of using confocal laser is that it can focus Laser right above the nitrocellulose coating. Integrated software is used to automatically analyze array images, qualify and quantify spots in series, and generate serial dilution curves to determine the relative protein levels and phosphorylation states in the samples.

To demonstrate the capacity of our platform to capture the dynamics of signaling responses, and determine the sensitivity to detect minute changes, glioma cancer cells expressing constitutively activated EGFRvIII mutant under tetracycline control were analyzed by protein arrays. The EGFRvIII mutant is a common oncogenic mutant coexpressed with wild-type EGFR in glioblastoma (GBM) [12]. EGFRvIII is unable to bind ligand and signals constitutively. Kinetics of signaling after conditional induction of EGFRvIII expression was analyzed to quantify the response. The dynamics of pathway interactions (i.e. cross-talks) between EGFR pathways and other signaling pathways were then captured.

\section{Results and discussion}

Understanding complex cellular systems will require the identification and analysis of each of its components and allow determination of how they function together and are regulated. A critical step in this process is to determine the biochemical activities of the proteins and how these activities themselves are controlled and modified by other proteins. Traditionally, the biochemical activities of proteins have been elucidated by studying single molecules, one experiment at a time. This process is not optimal, as it is slow and labor intensive. To obtain a global view of molecular events instead of individual molecules, we utilize the protein array approach to monitor the molecular network response.

\section{Linear Dynamics for Quantization}

To test the linearity of Qdot signal, we produced cell lysates with artificial gradient of $\mathrm{p} 53$ protein by mixing null p53 cell lysate (human lung cancer H358 cells with null p53 gene) and p53 mutant cell lysate (human lung cancer H2009 cells with overexpressing mutant p53) in different proportions. Figure $1 \mathrm{~A}$ is the array image amplified by confocal Laser scanner. Figure $1 \mathrm{~B}$ is the array image amplified by CSA (HRP-based) system. As shown in Figure $1 C, R 2$ value of the Laser-amplified linear regression curve is close to 1 indicating Qdot-RPPA can distinguish at least $25 \%$ change of protein levels within the linear range, verse the signals of CSA-amplification showing the sigmoid response indicating lost of the linearity.

\section{Sensitivity}

In Figure 2, using purified Akt protein, Qdot-RPPA, like CSA-RPPA, can detect as low as $0.1 \mathrm{pg}$ compared to $0.1 \mathrm{ng}$ of the detection limit using traditional Western blot in normal conditions, at least 1000-fold more sensitive (Figure 2A vs. C). Moreover, the signal linearity over serial dilutions makes Qdot-RPPA a reliable tool for quantification (Figure 2B vs. D).

\section{Specificity}

Qdot-RPPA can detect specific kinase activities with validated phospho-specific antibodies. In Figure 3, Qdot-RPPA distinguishes phospho-AKT (pAKT ser473) activation in the PI3 kinase inhibitor (LY294002) vs. phosphatase inhibitor (Calyculin A) treatments as well as with vs. without serum stimulation. Total AKT is not altered during treatment. Thus, with a pair of validated total and phospho-specific antibodies, Qdot-RPPA can be used to monitor functional status of a given kinase under different treatments, or diseases.

\section{Reproducibility}

The signals of Qdot are reliable over time. Examples of ERK protein stain are shown in Figure 4. The same samples were spotted and hybridized with Qdot at the different time in duplicate. $\mathrm{R}^{2}$ values are about 0.95 .

\section{Linearity Tests for Phospho-specific Antibodies and Caspase}

In Figure 5, a series of commercial available negative and positive controls for indicated phospho-specific and caspase 3 antibodies were mixed proportionally to generate artificial gradients of target proteins: 2A. pAKT; 2B. pERK; 2C. pGSK3; 2D. pNFkB; 2E. pp38; 2F. cleaved caspase 3. The intensities from $0 \%$ gradients were considered to be 


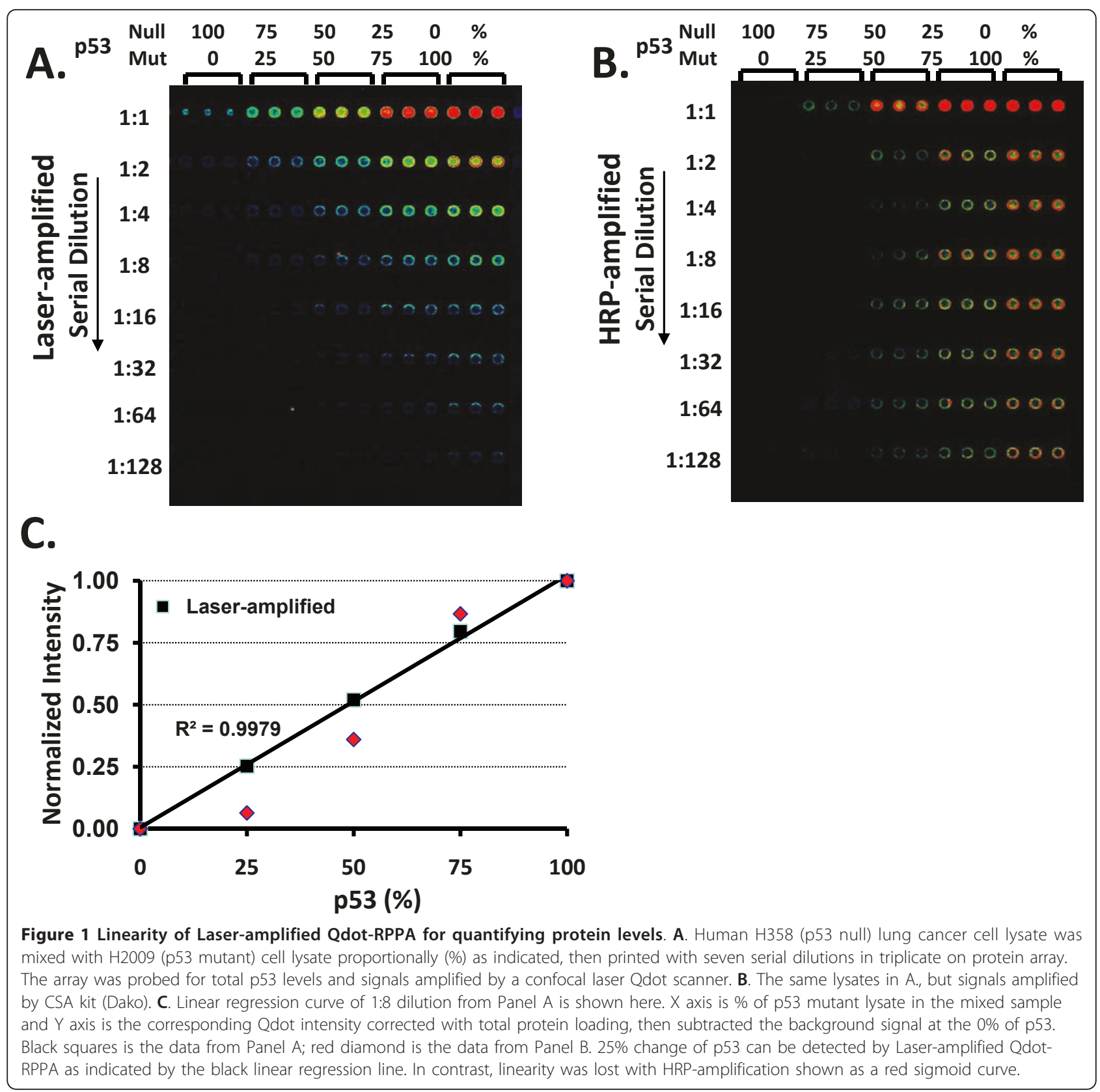

non-specific signals from the antibodies, and deducted from the readouts of other gradients. The $\mathrm{Y}$ axis is the calibrated signal intensity after subtracting the non-specific background signal. $R^{2}$ values of these Laser-amplified linear regression curves are near 1 assuring that QdotRPPA can distinguish at least $25 \%$ change of protein functional levels while using validated antibodies.

\section{Array Image Processing and Statistic Readouts}

Compared to commercially available DNA microarrays, protein arrays present additional challenges in image analysis. The variety of array formats, spot shapes, and intensity profiles makes it challenging to extract spot signals correctly. In addition, the different array substrates, printing mechanisms and protocols, staining/blocking processes, and broad applications result in various kinds of complex images. It is extremely difficult to develop one algorithm fit all applications. MicroVigene ${ }^{\mathrm{TM}}$ is implemented based on the object-oriented technology and enable a robust software system integrating with multiple algorithms that is flexible, configurable as well as extensible to provide customized solutions, support any future needs, and adapt along with this emerging field through proper plug-ins (Figure 6) [13]. 


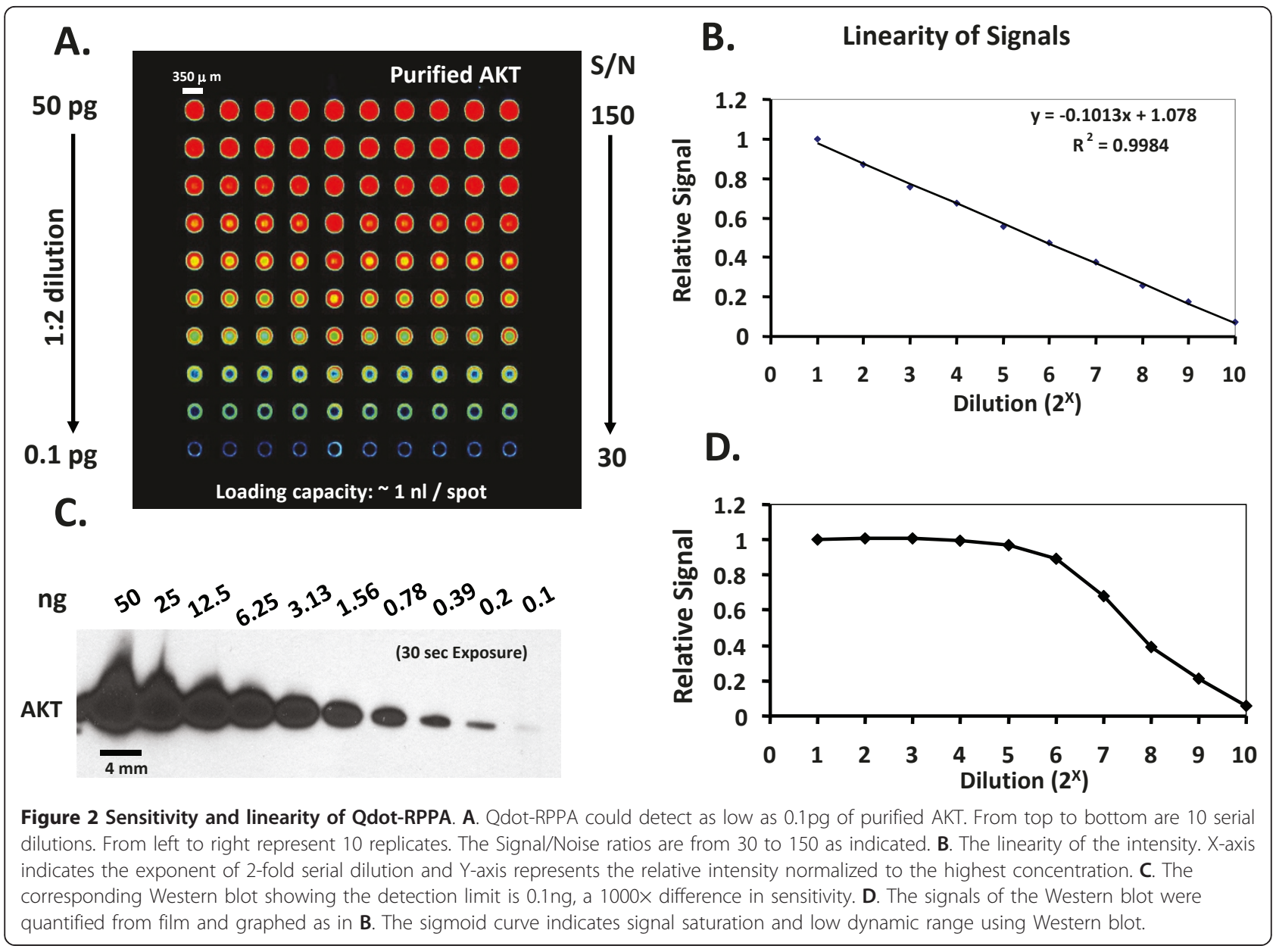

Customized MicroVigene ${ }^{\mathrm{TM}}$ microarray image analysis software has been developed for high throughput, automatic array image processing and quantitative readouts from serial dilutions of samples. The software implements the actual boundary algorithm for spot identification/segmentation that is resistant to the spot shift and image shift (Figure 7); the regional background algorithm for local non-uniform background correction and sensitive spot quantification (Figure 8).

Also, instead of generating multiple linear regression curves for data quantification over each series of serial dilutions, MicroVigene ${ }^{\mathrm{TM}}$ implements the SuperCurve algorithm (details in the Methods section) [14] that using all spots within one array to form a sigmoid antigen-antibody binding kinetic curve (i.e. SuperCurve) (Figure 9A). The advantage of SuperCurve is the resistance to the experimental outliers or missing spots on the array compared to regular linear regression curves formed by only a few spots over a serial dilution. The SuperCurve is a consensus curve supported by all spots on the array. This process has been implemented into the automatic data analysis workflow after the spot identification to automatically generate a reaction curve for each antibody as well as the quantitative readouts from each dilution series.

The automatic dust finding and removal algorithm is also implemented to increase the accuracy of curve fitting and the intensity readouts. In Figure 9B, a small but intensified dust spot was automatically detected in the lowest dilution spot, and then visualized by 3D image manually. Without the dust removal, the spot was flagged as an outlier of the SuperCurve verse, after removing the dust signal, the spot fitted in the SuperCurve well (Figure 9C).

In summary, these unique features of MicroVigene ${ }^{\mathrm{TM}}$ make it capable of handling shifted and noisy protein array images and enabling the hand-free batch process required for high throughput protein array image processing.

\section{The Dynamics of EGFRvIll Signaling Network}

Effective targeted treatment of glioblastoma (GBM) may require a coordinated inhibition of multiple signals. Much remains to be learned about functional interactions among signal transduction networks in GBM. For 

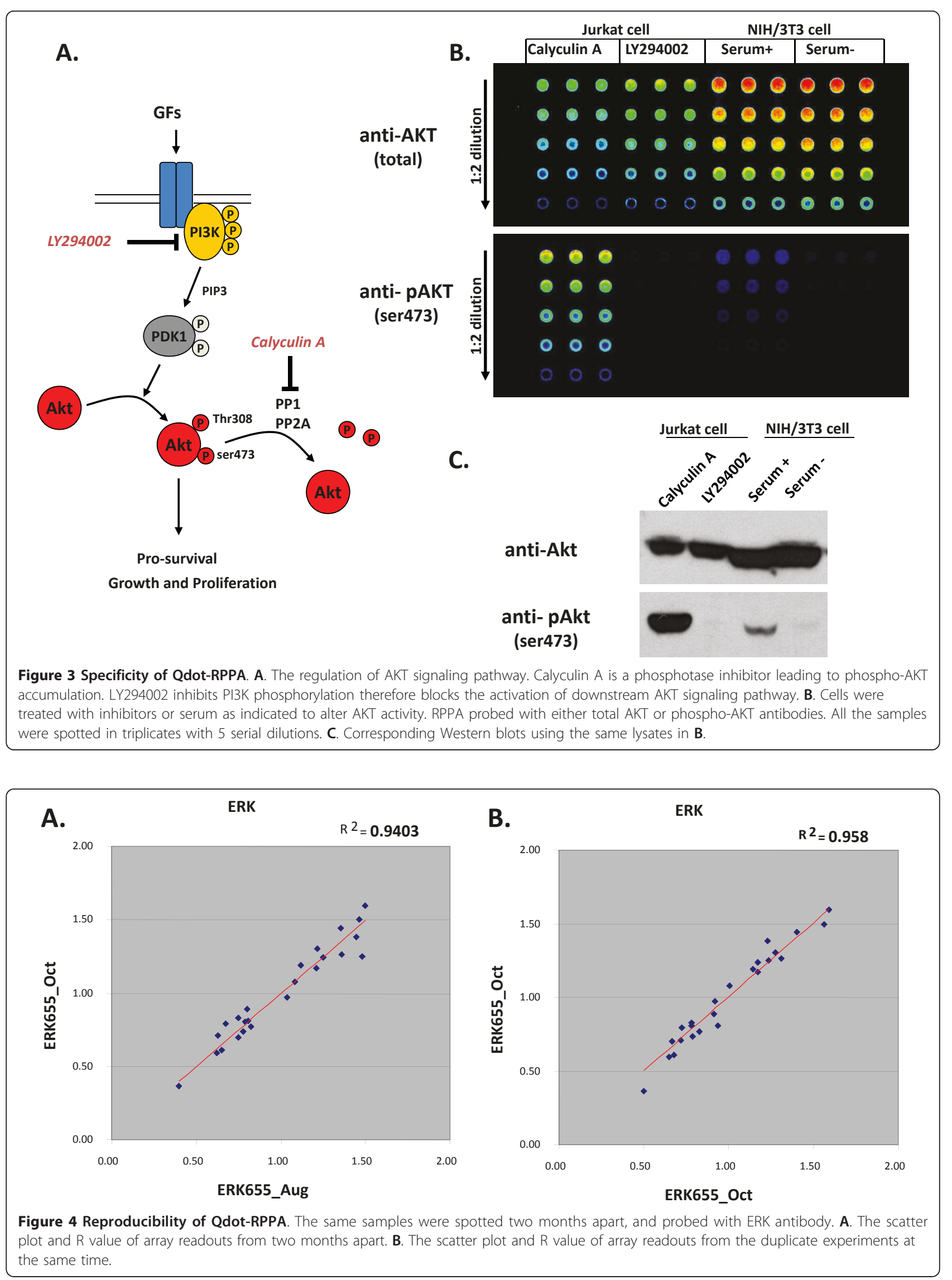


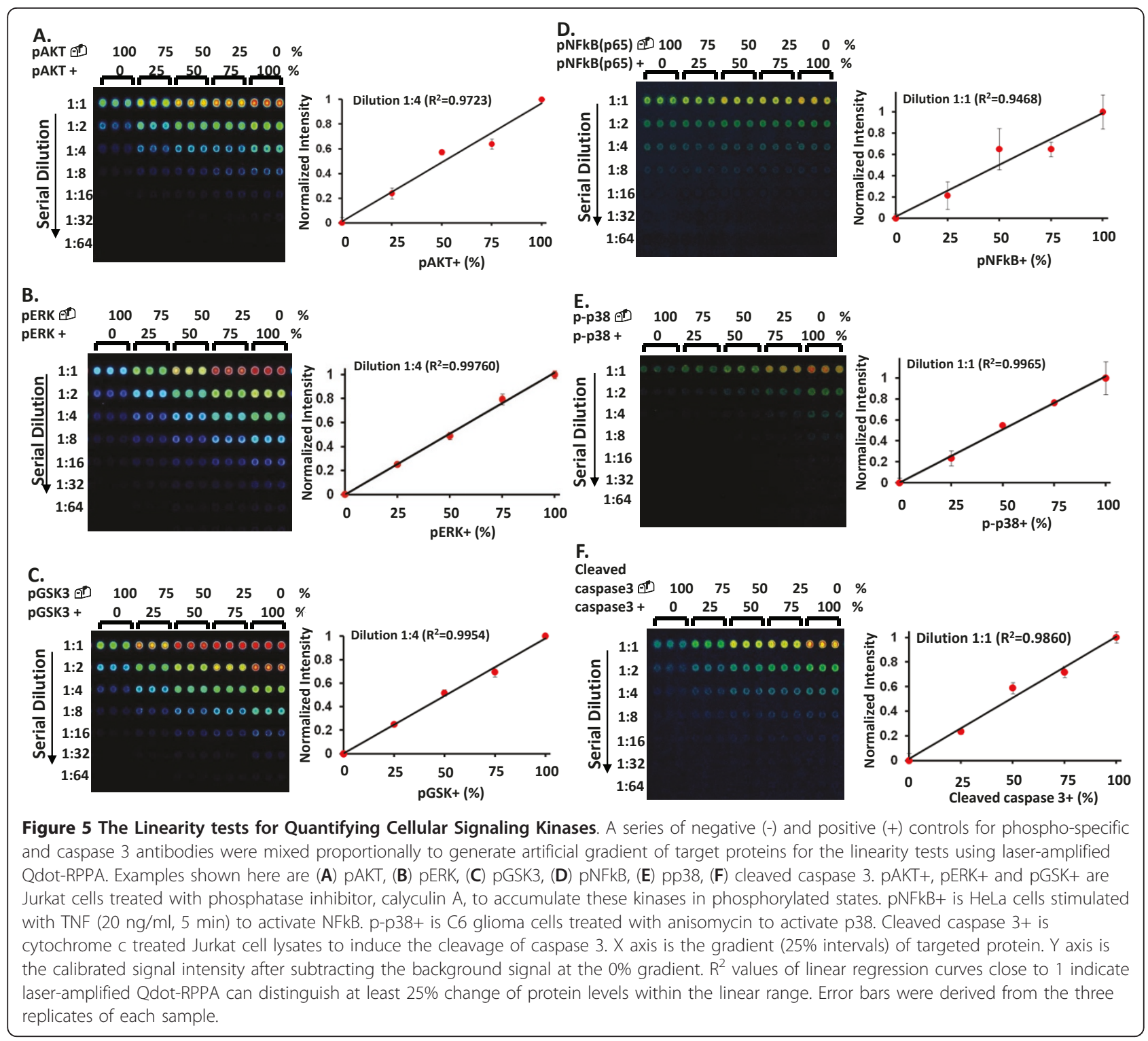

example, GBMs express multiple receptor tyrosine kinase (RTK) families and ligands. Increasing evidence suggest extensive cross-talking between RTK signaling networks that have functional implications for multi-target treatment. EGFRvIII is a common oncogenic mutant that is co-expressed with the wild-type EGFR in GBM. We applied Qdot-RPPA to investigate key protein regulators and kinases (total 61 antibodies listed Table 1) that are altered by conditional inducible EGFRvIII in U87MG glioma cell line. The dynamics of pathway interactions (i.e. cross-talks) among canonical EGFR pathway, Akt, Src and JNK pathways after turning on the EGFR vIII were captured (Figure 10). Qdot-RPPA detected the total EGFR, pEGFR and pERK levels changes within one to six hours after adding tetracycline; Activation of Akt $[15,16]$, Src $[17,18]$ and JNK
[19-21] happened at later time (20-24 hrs), and, interestingly, pSrc $(52.8 \times)$ and pJNK $(4.4 \times)$ were activated at much higher levels than the downstream pERK $(3.8 \times)$ in the canonical pathway (Array images shown in Figure 10 and fold change in activity shown in Table 2). The results suggest the selection of potential candidates for the future multi-target treatment in GBM, e.g. co-targeting EGFRvIII and Src/JNK kinases for cancer treatment $[17,18]$ since Src and JNK activities are elevated much higher than ERK over time.

\section{Conclusions}

In conclusion, we have established a quantitative proteomic technology - Qdot-RPPA platform, in conjunction with the emerging Quantum dot (Qdot) nanotechnology, a confocal laser scanner, computer automated array image 


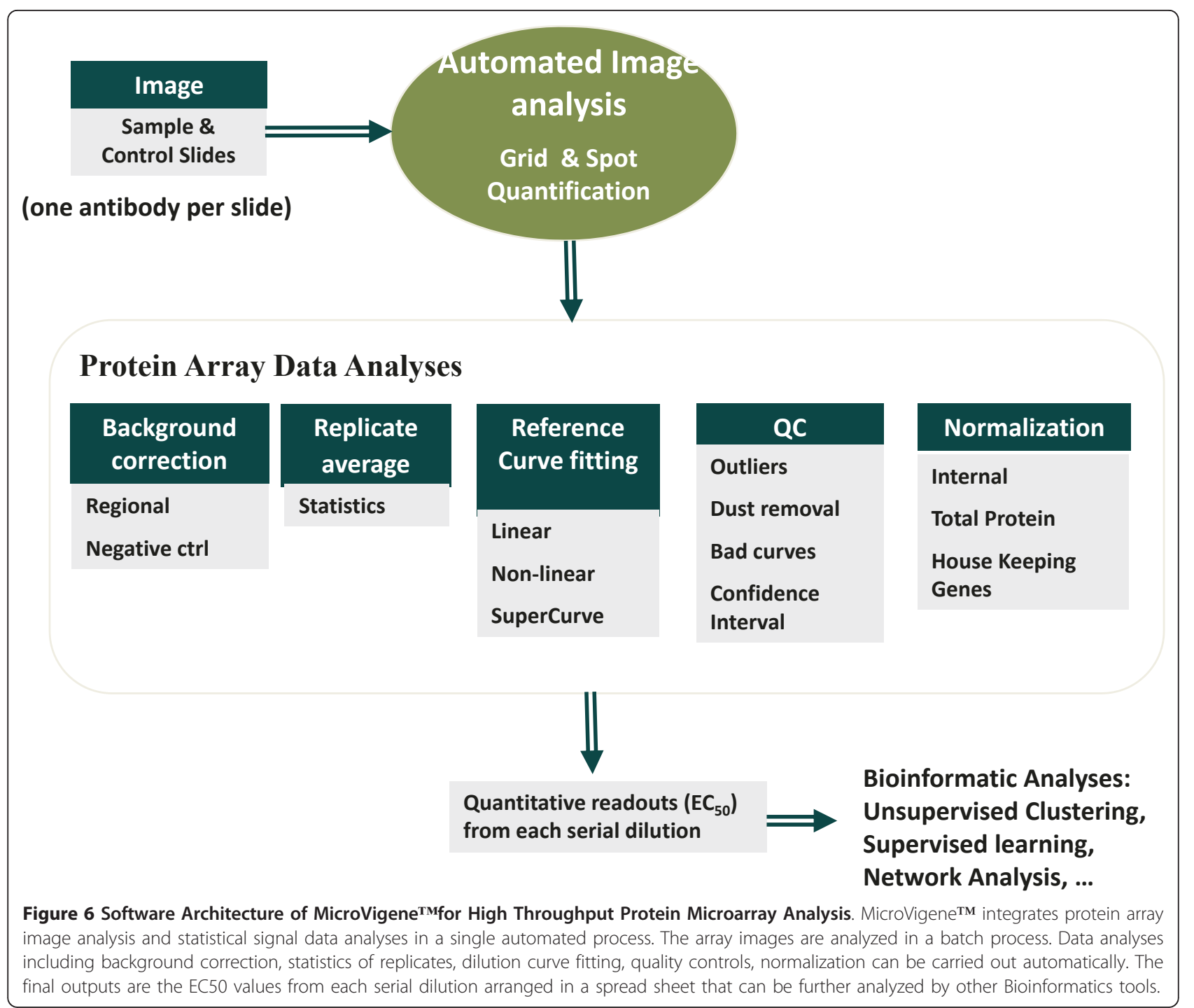

analysis to generate quantitative outputs. This platform eliminates the signal non-linearity causing by enzymeamplification, while still keeps the advantages of RPPA such as: minute sample per $\operatorname{spot}(\sim 1 \mathrm{nl})$; high sensitivity ( $\sim 0.1 \mathrm{pg})$; high specificity with validated antibodies; high reproducibility; and large capacity of spots on one slide allowing investigating a large number of experimental conditions in parallel within one array.

The primary contribution of our Qdot-RPPA platform is to take advantage of Qdot linear signal with no photobleaching and large dynamic range for quantification by utilizing confocal Laser Qdot scanner. This technology not only offers us the capacity to quantitatively monitor the time series and dose responses of cellular response over signaling network after treatments or different disease stages, but also facilitates the complex functional analysis among different signaling pathways. We also found that thin coated (10 um) nitrocellulose slide with scanning Laser focus above the coating yields low fluorescence background and increases signal/noise ratio.

We have applied Qdot-RPPA platform to study the Dynamics of EGFRvIII Signaling Network with 61 antibodies. The results demonstrated this platform worked well to reveal not only the dynamics of canonical pathway but also cross-talks among pathways. The ultimate goal is to extend this platform with more validated antibodies to provide high sample throughput functional protein data to compliment DNA based genomics array data for systems biology analyses, and provide direction for more in-depth experimentation prediction and hypothesis generation.

\section{Methods}

Cell Lysate Preparation

p53 control cell lysates were prepared from human H358 (p53 null) lung cancer cells and H2009 (p53 mutant) cells. The rest of control cell extracts were purchased 


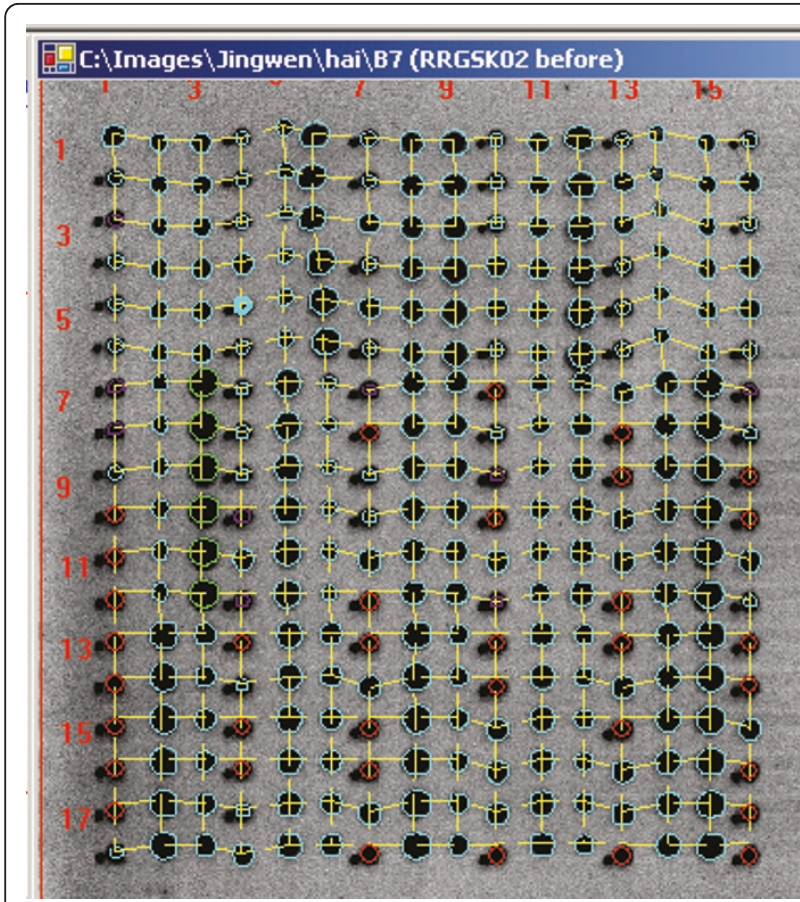

Figure 7 Actual Boundary and Flexible Grid Algorithms. Image and spot shifts among arrays are the major challenge for the automation of array image processing. The fixed spot grid cannot be applied to all images. The actual boundary and flexible grid algorithms are designed to make spot boundary and grid flexible enough to compensate the shifts. These algorithms are essential for the successful automation of array image analysis using MicroVigene ${ }^{\mathrm{TM}}$. from Cell Signaling Technology: Akt (cat\# 9273), p38 MAP kinase (cat\# 9213), NF- $\kappa$ B (cat\# 9243), and caspase-3 (cat\# 9663). Purified Akt protein was purchased from Invitrogen (cat\# P2999). U87MG glioma cells were stably transfected with an EGFRvIII mutant using a tetracycline inducible system as described previously [22]. Cells were treated with tetracycline $(1 \mathrm{ug} / \mathrm{ml})$, and then, harvested at 0, 6, 20, 24 and 48 hours, respectively. The following cell lysis protocol is optimized to harvest total cell proteins including hard-to-dissolved membrane proteins without using Urea. Cells were washed twice in cold phosphate buffered saline (PBS) and subsequently lysed in 250-350 $\mu \mathrm{L}$ hot lysis buffer (2\% SDS; $0.06 \mathrm{M}$ Tris-Cl, pH 6.8; 5\% Glycerol). Proteinase inhibitor (Sigma, cat\# P8340) and phosphotase inhibitor cocktails (Santa Cruz Biotechnology, sc-45044 and sc-45045) and 2\% $\beta$-mercaptoethanol were freshly added before use. The cellular lysates were boiled for $5 \mathrm{~min}$ on heat block, followed by 1 min vortexing, and then centrifuged at 13,000 rpm for $7 \mathrm{~min}$ at $4^{\circ} \mathrm{C}$. Cells should be completely dissolved without much precipitates left at this step. Supernatants were transferred to new tubes and stored at $-20^{\circ} \mathrm{C}$. Protein concentration was measured using Bradford assay (Bio-Rad, cat\# 500-0006). All the samples were adjusted to $0.5 \mathrm{ug} / \mathrm{uL}$ as the highest concentration on the arrays. However, to correct for protein loading, Sypro Ruby ${ }^{\mathrm{TM}}$ protein stain signals were used as described below.

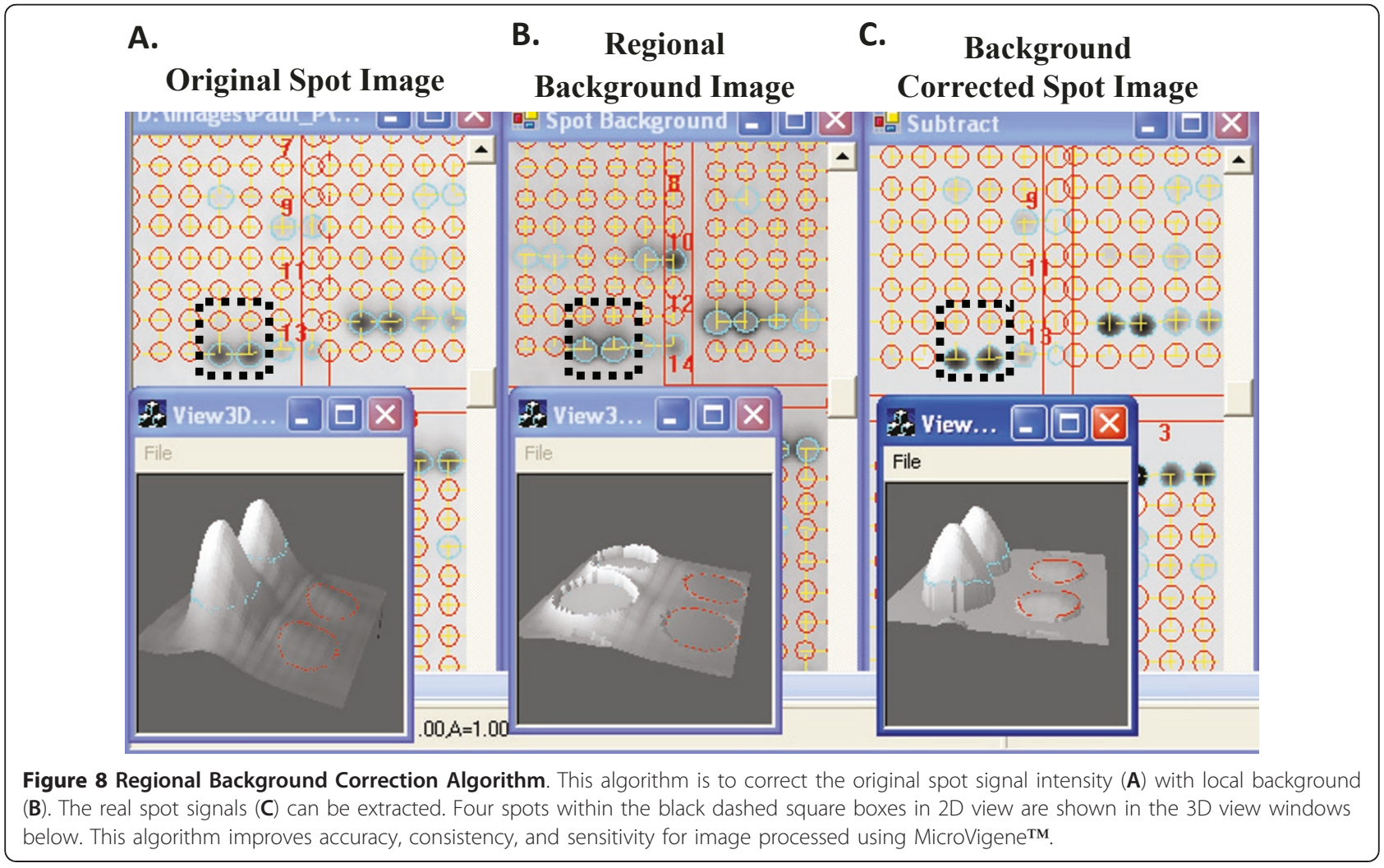




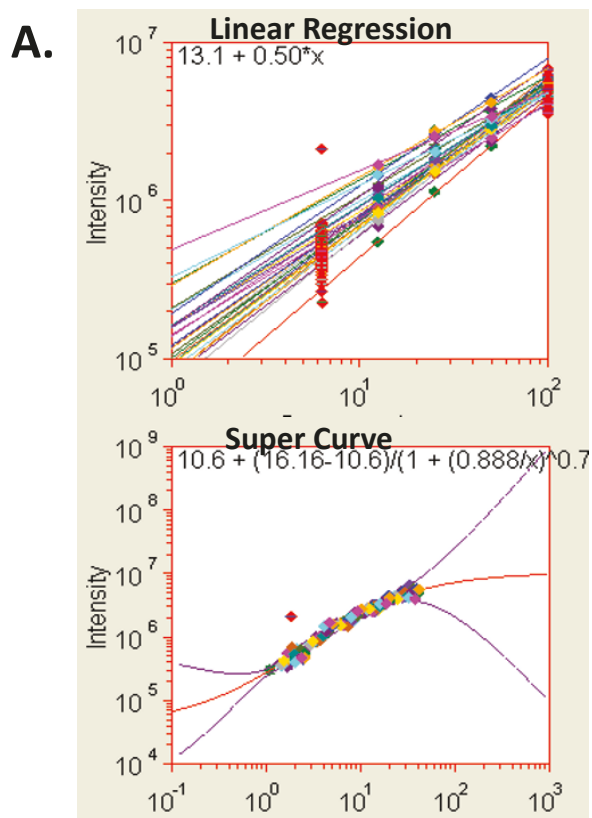

\section{Before dust removal}

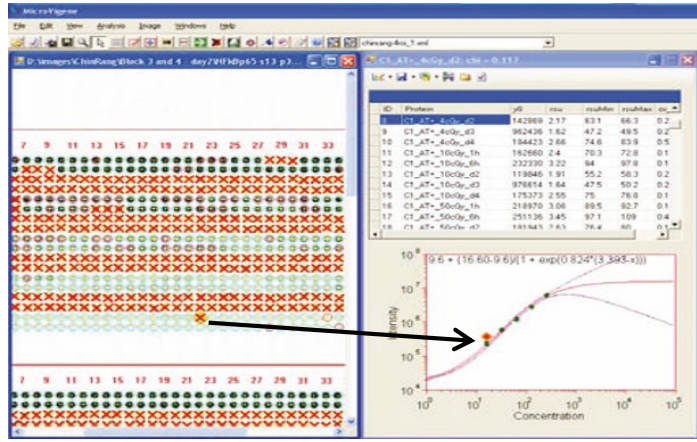

B.
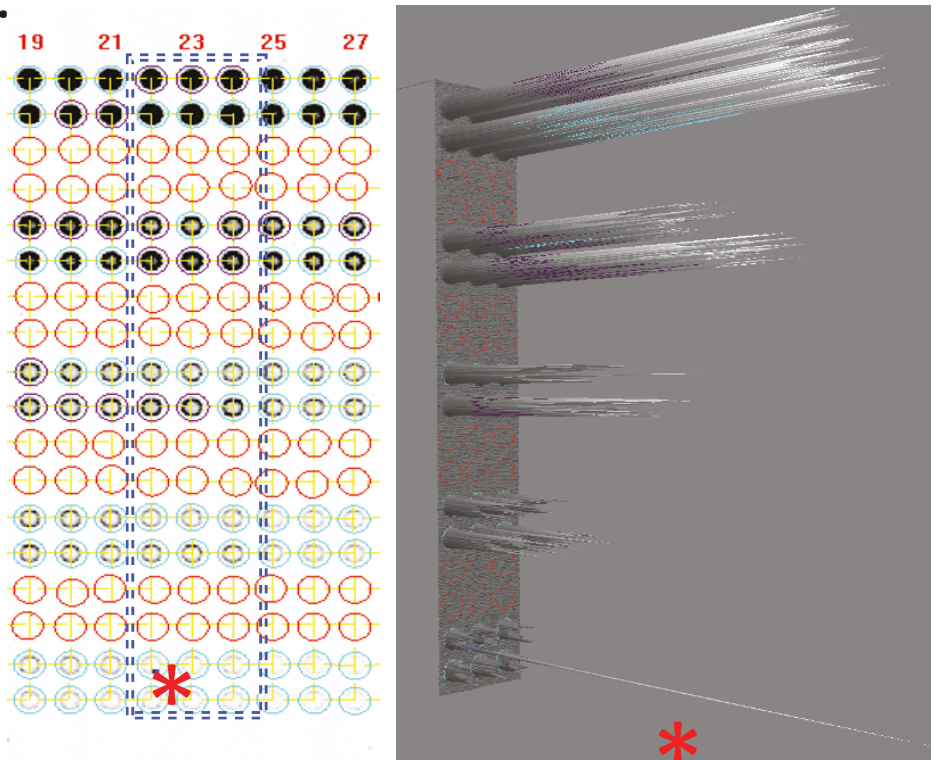

After

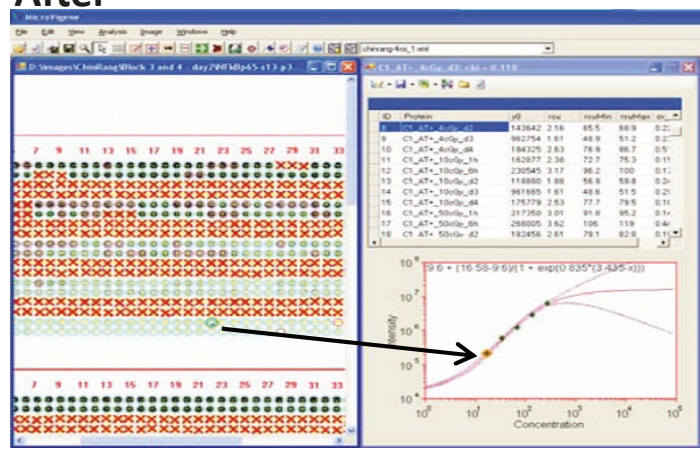

Figure 9 Automation of Qdot-RPPA Image and Data Analysis. Several features of MicroVigene ${ }^{\mathrm{TM}}$ software are to facilitate the automatic batch process. A. Super Curve: Instead of generating multiple linear regression curves for each serial dilution, the Super Curve algorithm uses all spots on the same array to fit one antigen-antibody reaction curve (called Super Curve, the red line) for the quantitative readouts. The purple lines indicate error boundary. B. 2D and 3D views of the actual spot boundary algorithm for actual spot identification and dust finding (indicated by asterisk); the total intensity within the defined spot boundary (i.e. volume) was used for Super Curve fitting. C. Screen shots of the dust removal feature. Left: before removing the dust, the spot is flagged as an outlier (the red spot pointed by the arrow). Right: after dust removal, the spot fits the Super Curve.

\section{Qdot-Reverse-Phase Protein Microarray (Qdot-RPPA)}

Protein lysates were filtered through 96-well filter plate with $25 \mu \mathrm{m}$ pore membrane (Phenix Research Products, cat\# MPF-009) by centrifuge to remove sticky aggregates, and serially diluted (1:2) 4 times using $1 \times$ lysis buffer. Lysates were arrayed on ONCYTE ${ }^{\circledR}$ AVID nitrocellulose film slides (Grace Bio-Labs, cat\# 305170) using a SpotArray $^{\mathrm{TM}} 24$ Microarray printing system (PerkinElmer) with 55-60\% humidity. Spots were separated with $350 \mu \mathrm{m}$ space in between. Approximately $1 \mathrm{nl}$ of lysate per spot was arrayed using 4 spotting pins (TeleChem/ArrayIt, cat\# 946MP3). Slides were dried at RT for about $30 \mathrm{~min}$, and then stored at $4^{\circ} \mathrm{C}$. Immuno-staining was performed within one week. We have tested various slides from different vendors. The AVID slides were chosen due to its high protein binding capacity with less protein lost, and thin-coated (10 micron) nitrocellulose film with less auto-fluorescence background, suitable for both Qdot ${ }^{\mathrm{TM}}$ and SyproRuby ${ }^{\mathrm{TM}}$ stains.

\section{Laser-amplified Qdot-RPPA and Immuno-staining}

The slides were placed into four-chamber plates (ISC Bioexpress, cat\# T-2896-1) and incubated at room temperature in Re-blot Plus Mild Solution (CHEMICON, cat\# 2502) for no more than 7 minutes to relax protein structure. After the Re-blot was removed, the slides were washed $5 \mathrm{~min}$ three times in TBS-T buffer (2.42 g Tris-

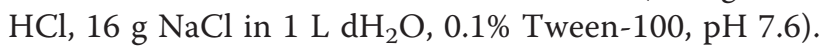




\begin{tabular}{|c|c|c|}
\hline AKT & $\mathrm{Rb}$ & NFkBp65 \\
\hline pAKT(S473) & pRb(S807_811) & pNFkBp65(S536) \\
\hline pAKT(T308) & p16 & $\mathrm{IKBa}$ \\
\hline ERK1/2 & p21 & plKBa(S32) \\
\hline pERK1/2(S44/42) & p27 & MDM2 \\
\hline GSK3ab & CyclinB1 & pMDM2(S166) \\
\hline pGSK3ab(S21/9) & CyclinD3 & E-Cadherin \\
\hline IRS1 & Cdk4 & Vimentin \\
\hline pIRS1(Y896) & EGFR & b-Catenin \\
\hline pIRS1(Y1179) & pEGFR(Y1173) & $\mathrm{gH} 2 \mathrm{AX}$ \\
\hline mTOR & IGF1R & Hsp27 \\
\hline pmTOR(S2448) & plGF1R(Y1158_1162) & NQO1 \\
\hline PTEN & plGF1R(Y1162_1163) & sClu \\
\hline pPTEN(S380) & Raf1 & b-Actin \\
\hline p38 & pRaf1(S259) & Smad3 \\
\hline pp38(T180_Y182) & $\mathrm{BCl} 2$ & pSmad3(S423/425) \\
\hline SrC & $\mathrm{pBCl}-2(\mathrm{~S} 70)$ & Cleaved Caspase 3 \\
\hline $\mathrm{pSrc}\left(\mathrm{Y}_{416)}\right.$ & JNK & Cleaved Caspase 8 \\
\hline pSrc(Y527) & pJNK(T183_185) & Cleaved Caspase 9 \\
\hline Stat3 & p53 & \\
\hline pStat3(S727) & pp53(\$15) & \\
\hline
\end{tabular}

The slides were incubated in Sea Block blocking buffer (Thermo, cat\# 37527) at $4^{\circ} \mathrm{C}$ overnight, and then blocked with Avidin and Biotin (Dako, Biotin blocking system cat\# X0590) sequentially. Between steps, slides were washed with TBS-T buffer. After the blocking, the slides were incubated with primary antibodies (diluted in antibody diluent buffer, Dako, cat\# S3022) at $4^{\circ} \mathrm{C}$ overnight. We used 61 validated primary antibodies listed in the Table 1 and the Additional file 1; validation information is in [3]. Next, slides were washed with TBS-T buffer, and incubated with biotinylated secondary antibodies (Vector, antirabbit BA-1000 or anti-mouse BA-9200 IgG) (1:5000) for $30 \mathrm{~min}$, then with Qdot 655-streptavidin conjugate (Invitrogen, cat\# Q10121MP) for $30 \mathrm{~min}$. Qdot were diluted 1:200 in boric acid buffer ( $50 \mathrm{mM}$ Borate, $2 \% \mathrm{BSA}, \mathrm{pH}$ 8.3). The slides were washed with TBS-T buffer 3 times, and MilliQ water once and then briefly spun at $2000 \mathrm{rpm}$ for $5 \mathrm{~min}$ to dry the slides. To detect Qdot signals, slides were scanned with full Laser power on a ProScanArray Microarray Scanner (PerkinElmer), and the Laser was focused 10 micron above the slide surface. The excitation wavelength was set at $488 \mathrm{~nm}$ and emission wavelength was set at $655 \mathrm{~nm}$ using a Qdot 655 filter. Images were saved in 16-bit TIFF format and the maximum signal intensity was 65535 .

\section{Enzyme-based Qdot-RPPA}

Qdot-RPPA using the Tyramide and horseradish peroxidase (HRP) amplification system (Dako CSA ${ }^{\mathrm{TM}}$ system, cat\# K1500) to amplify the Qdot signal was previously described $[5,6]$. This protocol was performed for the comparison shown in Figure 1B.

\section{Automatic Array Image analysis and Quantification}

Scanned TIFF images were batch-analyzed using MicroVigene $^{\mathrm{TM}}$ software (VigeneTech inc. http://www.vigenetech. com/). In RPPA, cell lysates were printed on the arrays. Each array is probed with one specific primary antibody and scanned for one image. It is necessary to have computer-aid image analysis that can process array images automatically to increase the throughput of the number of antibodies one can apply. This work was done in collaboration with VigeneTech, Inc., a leader of developing automated image analysis technology. MicroVigene ${ }^{\mathrm{TM}}$ provided unique software customized to analyze the QdotRPPA platform. Unique features of the software provide accuracy, sensitivity, and reliable results of automation [13] including flexible grid and actual spot boundary algorithm to quantify spot signals accurately; dust removal algorithm to remove the contaminated signals; regional background algorithm for local non-uniform background correction and sensitive spot quantification. Ten thousand spots can be processed in less than one minute and, with the feature of hands-free batch processing, MicroVigene ${ }^{\mathrm{TM}}$ enables the high throughput protein array image analysis. $3 \mathrm{D}$ visualization of processed images is also available for manually quality assurance.

Each sample has five dilutions and was printed in triplicate on the array. Therefore, each dilution series has total of 15 data points to minimize errors and increase the confidence of curve-fitting. Instead of generating multiple linear regression curves for data quantification over each series of serial dilutions, MicroVigene implements the SuperCurve algorithm (a 4-parameter logistic-log model, i.e. parameters a-d shown in equation below) that uses all spots within one array to form a sigmoid antigen-antibody binding kinetic curve (i.e. SuperCurve) [14].

$$
Y=a+\frac{(b-a)}{\left(1+e^{\left(c^{*}(d-\ln (x))\right)}\right)}
$$

where $\mathrm{x}$ is the dilution factor and $\mathrm{Y}$ is the signal intensity. The signal readout of each dilution series is the intensity of EC50 from the fitted SuperCurve. The assumption is that the same antibody-antigen binding kinetics is taking place at each sample spot, even in the different samples, thus by taking all spots on an array to fit a common response curve can increase the confidence of the curve fitting.

Calibration for protein loading was based on total protein per spot. For estimation of total protein amounts, randomly selected arrays were stained with Sypro 


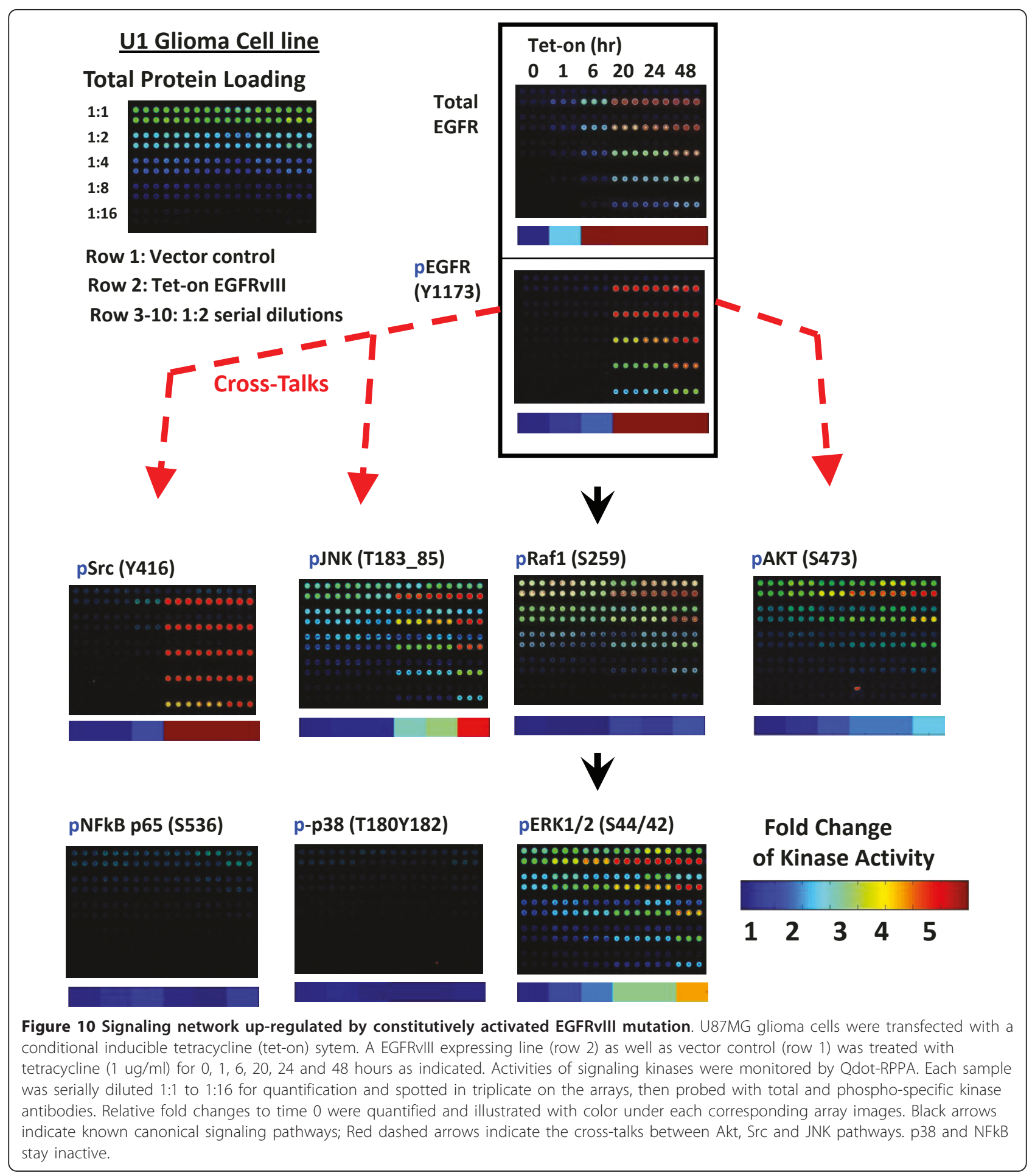

Ruby $^{\mathrm{TM}}$ (Invitrogen, cat\# S11791), and visualized on ProScanArray Scanner (PerkinElmer) with excitation wavelength at $450 \mathrm{~nm}$ and emission wavelength at 610 $\mathrm{nm}$. The corrected values were calculated by dividing the EC50 readouts of the antibody to the corresponding EC50 readouts of the Sypro Ruby ${ }^{\mathrm{TM}}$ stain. In Figure 5, the $0 \%$ positive gradient on the left was considered as a non-specific signal from the antibody, and deducted from the readouts of other gradients. In Figure 10, the intensity of time point 0 was treated as a baseline and relative fold change of activity at the other time points were compared to time 0 . 
Table 2 Fold Change of Signaling Kinases Up-regulated by Constitutively Activated EGFRvIII Mutation over Time

\begin{tabular}{lccccccccc}
\hline Time & EGFR & $p E G F R(Y 1173)$ & $p$ Raf1 & $p$ Erk & $p$ Akt & $p$ Src(Y416) & $p J N K$ & $p p 38$ & $p N F-k B p 65$ \\
$0 \mathrm{hr}$ & 1.00 & 1.00 & 1.00 & 1.00 & 1.00 & 1.00 & 1.00 & 1.00 & 1.00 \\
$1 \mathrm{hr}$ & 2.28 & 1.14 & 0.88 & 1.36 & 1.08 & 0.99 & 1.02 & 1.01 & 1.08 \\
$6 \mathrm{hr}$ & 7.31 & 1.75 & 0.89 & 1.89 & 1.62 & 1.51 & 1.04 & 0.91 & 1.24 \\
$20 \mathrm{hr}$ & 23.19 & 26.19 & 1.32 & 2.76 & 1.88 & 31.35 & 2.61 & 0.93 & 0.95 \\
$24 \mathrm{hr}$ & 22.14 & 25.11 & 1.17 & 2.77 & 1.88 & 34.39 & 2.82 & 0.88 & 0.92 \\
$48 \mathrm{hr}$ & 26.39 & 35.62 & 1.59 & 3.79 & 2.26 & 52.79 & 4.37 & 0.99 & 1.26 \\
\hline
\end{tabular}

\section{Additional material}

Additional file 1: Antibodies used for Qdot-Reverse Phase Protein

Array. Detail information of antibodies used.

\section{List of abbreviations}

CSA: Catalyzed Signal Amplification; GBM: glioblastoma; HRP: horseradish peroxidase; Qdot: Quantum dot; RPPA: Reverse phase protein lysate array; RTK: receptor tyrosine kinase; TSA: Tyramide Signal Amplification.

\section{Acknowledgements}

We thank Drs. Kevin Rosenblatt, Jean Gao and Mike Story for their consultations and suggestions; Dr. Alan Miller for proof-reading the manuscript. This research was supported by the Office of Science, U.S. Department of Energy, Grant No. DE-FG02-07ER64335 (C-R.Y.) and by DEFG02-09ER64789 (DAB). This work was also supported by grants from the National Institutes of Health Grant (R01AG019712) (MK), and R01NS062080 $(\mathrm{AH})$. We also thank the support from NIH/NCl Cancer Center Grant (1P30CA142543-01) to the Genomics and Protein Lysate Array Shared Resource. This is manuscript CSCN065 from the 'Cell Stress and Cancer Nanomedicine' program in the Simmons Comprehensive Cancer Center at the University of Texas Southwestern Medical Center at Dallas.

\section{Author details}

${ }^{1}$ Harold C. Simmons Comprehensive Cancer Center, University of Texas Southwestern Medical Center at Dallas, Dallas, TX 75390, USA. ²Department of Radiation Oncology, University of Texas Southwestern Medical Center at Dallas, Dallas, TX 75390, USA. ${ }^{3}$ Department of Pharmacology, University of Texas Southwestern Medical Center at Dallas, Dallas, TX 75390, USA. ${ }^{4}$ Department of Pathology, University of Texas Southwestern Medical Center at Dallas, Dallas, TX 75390, USA. ${ }^{5}$ Department of Neurology and Neurotherapeutics, University of Texas Southwestern Medical Center at Dallas, TX 75390, USA. ${ }^{6}$ North Texas VA Healthcare System, Dallas, TX 75216, USA. `VigeneTech, Inc. Carlisle, MA 01741, USA.

\section{Authors' contributions}

XW carried out the array experiments, protocol development, array image and data analysis, figures and manuscript preparation. YD, JP, MK participated in the early development of the protein array technology, provide personal expertise, protocols, and hand-on training to XW. YZ developed and test cell lysis buffers suitable for protein array printing. AJ, $\mathrm{AH}$ developed and provided EGFRvIII cell lines for protein array analyses. MR carried out software development to integrate the array image and data analyses. DB and CY conceived of overall experimental design, coordination among research groups, and manuscript preparation. All authors read and approved the final manuscript.

\section{Competing interests}

M.R. serves as CEO of VigeneTech, inc. In this study, the MicroVigene software was provided by M.R. based on collaboration. Other authors declare no competing interests

Received: 21 April 2011 Accepted: 15 September 2011 Published: 15 September 2011

\section{References}

1. Liotta LA, Espina V, Mehta Al, Calvert V, Rosenblatt K, Geho D, Munson PJ, Young L, Wulfkuhle J, Petricoin EF: Protein microarrays: meeting analytical challenges for clinical applications. Cancer Cell 2003, 3:317-325.

2. Spurrier B, Ramalingam S, Nishizuka S: Reverse-phase protein lysate microarrays for cell signaling analysis. Nat Protoc 2008, 3:1796-1808.

3. Kornblau SM, Tibes R, Qiu YH, Chen W, Kantarjian HM, Andreeff M, Coombes KR, Mills GB: Functional proteomic profiling of AML predicts response and survival. Blood 2009, 113:154-164.

4. Brase JC, Mannsperger H, Frohlich H, Gade S, Schmidt C, Wiemann S, Beissbarth $T$, Schlomm T, Sultmann $H$, Korf $U$ : Increasing the sensitivity of reverse phase protein arrays by antibody-mediated signal amplification. Proteome Sci 8:36.

5. Shingyoji M, Gerion D, Pinkel D, Gray JW, Chen F: Quantum dots-based reverse phase protein microarray. Talanta 2005, 67:472-478.

6. Geho D, Lahar N, Gurnani P, Huebschman M, Herrmann P, Espina V, Shi A, Wulfkuhle J, Garner H, Petricoin E, et al: Pegylated, steptavidin-conjugated quantum dots are effective detection elements for reverse-phase protein microarrays. Bioconjug Chem 2005, 16:559-566.

7. Sheehan KM, Calvert VS, Kay EW, Lu Y, Fishman D, Espina V, Aquino J, Speer R, Araujo R, Mills GB, et al: Use of reverse phase protein microarrays and reference standard development for molecular network analysis of metastatic ovarian carcinoma. Mol Cell Proteomics 2005, 4:346-355.

8. Sheehan KM, Gulmann C, Eichler GS, Weinstein JN, Barrett HL, Kay EW, Conroy RM, Liotta LA, Petricoin EF: Signal pathway profiling of epithelial and stromal compartments of colonic carcinoma reveals epithelialmesenchymal transition. Oncogene 2008, 27:323-331.

9. VanMeter AJ, Rodriguez AS, Bowman ED, Jen J, Harris CC, Deng J, Calvert VS, Silvestri A, Fredolini C, Chandhoke V, et al: Laser capture microdissection and protein microarray analysis of human non-small cell lung cancer: differential epidermal growth factor receptor (EGPR) phosphorylation events associated with mutated EGFR compared with wild type. Mol Cell Proteomics 2008, 7:1902-1924.

10. Wulfkuhle JD, Aquino JA, Calvert VS, Fishman DA, Coukos G, Liotta LA Petricoin EF: Signal pathway profiling of ovarian cancer from human tissue specimens using reverse-phase protein microarrays. Proteomics 2003, 3:2085-2090.

11. Loebke C, Sueltmann H, Schmidt C, Henjes F, Wiemann S, Poustka A, Korf U: Infrared-based protein detection arrays for quantitative proteomics. Proteomics 2007, 7:558-564

12. Hatanpaa KJ, Burma S, Zhao D, Habib AA: Epidermal growth factor receptor in glioma: signal transduction, neuropathology, imaging, and radioresistance. Neoplasia 2010, 12:675-684.

13. Ruan M: Protein Microarray Image Analysis. In Functional Protein Microarrays in Drug Discovery.. 1 edition. Edited by: Predki PF. CRC Press; 2006:359.

14. Hu J, He X, Baggerly KA, Coombes KR, Hennessy BT, Mills GB: Nonparametric quantification of protein lysate arrays. Bioinformatics 2007, 23:1986-1994.

15. Li L, Dutra A, Pak E, Labrie JE, Gerstein RM, Pandolfi PP, Recht LD, Ross AH: EGFRvIll expression and PTEN loss synergistically induce chromosomal instability and glial tumors. Neuro Oncol 2009, 11:9-21.

16. C VS, Babar SM, Song EJ, Oh E, Yoo YS: Kinetic analysis of the MAPK and PI3K/Akt signaling pathways. Mol Cells 2008, 25:397-406.

17. Andersen P, Villingshoj M, Poulsen HS, Stockhausen MT: Improved response by co-targeting EGFR/EGFRvIII and Src family kinases in human cancer cells. Cancer Invest 2009, 27:178-183. 
18. Bromann PA, Korkaya H, Courtneidge SA: The interplay between Src family kinases and receptor tyrosine kinases. Oncogene 2004, 23:7957-7968.

19. Antonyak MA, Kenyon LC, Godwin AK, James DC, Emlet DR, Okamoto I, Tnani M, Holgado-Madruga M, Moscatello DK, Wong AJ: Elevated JNK activation contributes to the pathogenesis of human brain tumors. Oncogene 2002, 21:5038-5046.

20. Antonyak MA, Moscatello DK, Wong AJ: Constitutive activation of c-Jun Nterminal kinase by a mutant epidermal growth factor receptor. $J$ Biol Chem 1998, 273:2817-2822.

21. Aikin R, Maysinger D, Rosenberg L: Cross-talk between phosphatidylinositol 3-kinase/AKT and c-jun NH2-terminal kinase mediates survival of isolated human islets. Endocrinology 2004 145:4522-4531.

22. Ramnarain DB, Park S, Lee DY, Hatanpaa KJ, Scoggin SO, Otu H, Libermann TA, Raisanen JM, Ashfaq R, Wong ET, et al: Differential gene expression analysis reveals generation of an autocrine loop by a mutant epidermal growth factor receptor in glioma cells. Cancer Res 2006 $66: 867-874$

doi:10.1186/1477-5956-9-53

Cite this article as: Wang et al:: Improved protein arrays for quantitative systems analysis of the dynamics of signaling pathway interactions. Proteome Science 2011 9:53.

\section{Submit your next manuscript to BioMed Central} and take full advantage of:

- Convenient online submission

- Thorough peer review

- No space constraints or color figure charges

- Immediate publication on acceptance

- Inclusion in PubMed, CAS, Scopus and Google Scholar

- Research which is freely available for redistribution

Submit your manuscript at www.biomedcentral.com/submit 